\title{
TÉCNICAS DE APRENDIZAJE PARA NIÑOS EN ETAPA PREESCOLAR CON DISLEXIA APLICANDO INTELIGENCIA ARTIFICIAL
}

\author{
John Gómez ${ }^{1}$, Javier Jadán², Mgs. Galo Hurtado³ \\ ${ }^{1}$ Institución \\ E-mail: jcgomez@sudamericano.edu.ec (DD https://orcid.org/0000-0002-0565-1932 \\ 2 Institución \\ E-mail: @ (D) https://orcid.org/0000-0003-1160-1437 \\ ${ }^{3}$ Institución \\ E-mail: gphurtado@sudamericano.edu.ec (D) https://orcid.org/0000-0002-7190-140X
}

Fecha de recepción: 08/07/2020

Fecha de aceptación: 03/02/2021

\section{RESUMEN}

El presente estudio analiza las técnicas de aprendizaje que pueden mejorar la formación de niños en etapa preescolar, el previo análisis pretende encontrar modelos y patrones que buscan de forma precisa como dar una solución de forma eficiente ante los problemas, conjuntamente con tecnologías que se encuentran en tendencia y su desarrollo es constante, con un campo aplicativo extenso, tal es el caso de la inteligencia artificial. El uso de tecnologías agiles no solo facilita un óptimo desarrollo en cuanto a la obtención de un producto cuyo objetivo es acaparar las expectativas y al mismo tiempo que cumpla la rama de investigación y todos los parámetros establecidos, en cuanto a la dislexia es un problema que dificulta el aprendizaje y el

\section{ABSTRACT}

The present study analyzes learning techniques that can improve the training of preschool children, the previous analysis aims to find models and patterns that seek precisely how to provide an efficient solution to problems, together with technologies that are on desarrollo de las capacidades lectoras y lingüísticas en niños de preescolar (4 a 6 años), por lo tanto, la implementación de ciertas tecnologías debe de acoplarse a las necesidades presentes en el sujeto. La parte aplicativa consta de un sitio web que busca ser interactivo mediante la aplicación de una evaluación de las capacidades intelectuales que determina si el niño presenta o no un trastorno disléxico. Luego de la prueba de inicio el sitio tendrá varios módulos en las cuales se podrá fortalecer las distintas capacidades y habilidades del usuario. La I.A cumple un rol fundamental durante este proceso ya que en base al análisis de respuestas e interacciones permitirá una mayor interactividad de la web y las funciones establecidas.

Palabras Clave: técnicas, desarrollo, software, tecnologías, dislexia, aprendizaje

trend and their development is constant, with an extensive application field, such is the case of artificial intelligence. The use of agile technologies not only facilitates optimal development in terms of obtaining a product whose objective is to hoard expectations and at the same time meets the research branch and all established parameters, in terms of dyslexia is a problem 
that hinders the learning and development of reading and linguistic skills in preschoolers (4 to 6 years), therefore, the implementation of certain technologies must be coupled with the needs present in the subject. The application part consists of a website that seeks to be interactive by applying an assessment of intellectual abilities that determines whether or not the child has a dyslexic disorder. After the start test the site will have several modules in which you can strengthen the different capabilities and skills of the user. The Artificial intelligence plays a fundamental role during this process because based on the analysis of responses and interactions will allow greater interactivity of the web and the established functions.

Keywords: techniques, development, software, technologies, dyslexia, learning

Forma sugerida de citar: Gómez, J., Jadán, J. \& Hurtado G. (2021). Técnicas de aprendizaje para niños en etapa preescolar con dislexia aplicando Inteligencia Artificial. Convergence Tech Revista Científica. 5(1), 30-37. https://doi. org/10.53592/convtech.v5iV.7

\section{INTRODUCCIÓN}

$\mathbf{E}$ 1 desarrollo de las capacidades lectoescritura en niños y niñas es primordial para el aprendizaje en el ámbito escolar, así como la adquisición de conocimientos.

\section{Según García [1]:}

La importancia de la lectura en los niños es tal que, sus beneficios se reflejan a la hora de estudiar y adquirir conocimientos. La colaboración de los padres es necesaria para impulsar el proceso de aprendizaje y para lograr que los niños se acerquen con gusto a los niños, para que, en definitiva, aprendan cuál es el placer de leer.

Las dificultades presentes en muchos niños al momento de leer o escribir ha sido un tema que ha llevado a realizar varias investigaciones con el objetivo de encontrar su origen y como solucionarlo.

La posibilidad que nos brindan las nuevas tecnologías con respecto a la solución de nuevos problemas son amplias y tienen una estructura fácil de manipular al mismo tiempo que nos permiten dar seguimiento a todos los resultados obtenidos que derivan de la interacción de entornos de aprendizaje.

Según el estudio realizado por la Universidad del Azuay (Vallejo, n.d.), Del total de 207 alumnos, 55 tenían algún tipo de dislexia. Concretamente, 20 alumnos tenían dislexia visual (36\%), 18 alumnos tenían dislexia fonológica (33\%) y 17 tenían dislexia mixta (31\%).

Para realizar los análisis que se detallan a continuación, se seleccionaron el grupo de desarrollo típico (DT) compuesto por 33 alumnos y los alumnos con algún tipo de dislexia (GD) que lo formaron 55 alumnos.

Por ellos los métodos y técnicas que se utilice para la reeducación de los niños con dislexia debe ser especializada y centrada en tratar dar a los infantes una atención personalizada en donde se evalué sus aptitudes y rendimiento en torno a su medio de aprendizaje y los avances que vaya logrando.

\section{INDICE DE LA DISLEXIA EN ECUADOR}

El ministerio de salud pública en la actualidad cuenta con un registro de 108.784 casos de discapacidad intelectual en Ecuador representados por grado de discapacidad y géneros. (Estadísticas de Discapacidad - Consejo Nacional Para La Igualdad de Discapacidades, n.d.)

Figura 1 » Grado de discapacidad-Ecuador

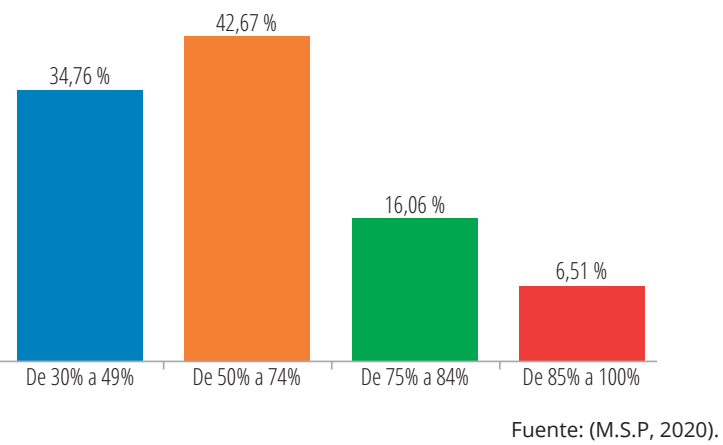

En la figura 1 se puede observar que el mayor grado de discapacidad pertenece entre un $50 \%$ a $74 \%$ con un porcentaje del $42.67 \%$ respecto a los 180.784 casos.

Los datos demográficos de géneros representan que un 54.86\% corresponde a masculino mientras que un $45.13 \%$ a femenino como se observa en la figura 2. 
Figura 2 » Géneros-Ecuador

Género

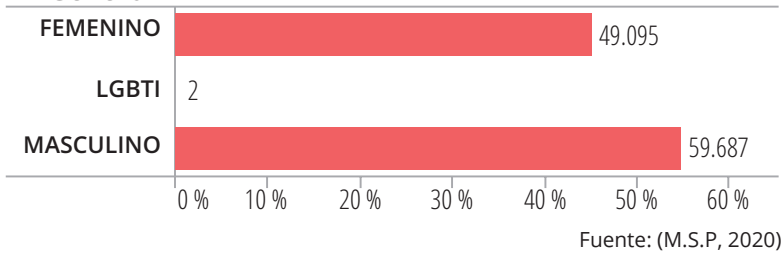

Centrándonos en nichos demográficos específicos respecto a la discapacidad disléxica tenemos el caso de Azuay con 6.257 personas que presenta un $5.75 \%$ con los siguientes datos en grado de discapacidad y géneros.

Azuay por su parte consta con un mayor grado de discapacidad entre $30 \%$ a $49 \%$ con un porcentaje del $48.46 \%$ respecto a los 6.257 casos.

Figura 3 » Grado de discapacidad-Azuay

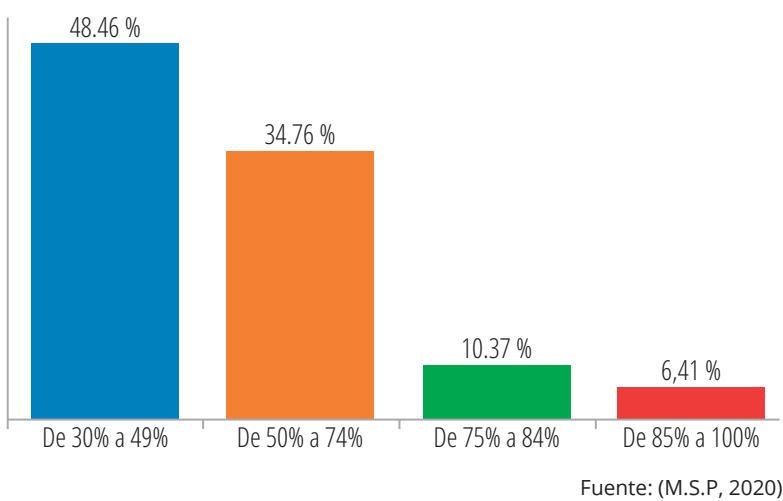

Figura 4 » Géneros-Azuay

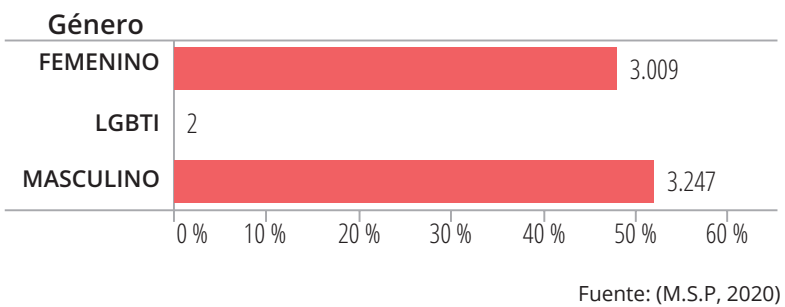

$\mathrm{Al}$ igual que en la Figura 2 la mayoría de personas con discapacidad dislexia pertenece al género masculino y en el caso de Azuay representan un 51.89\%

El Ministerio de salud pública nos presenta los porcentajes de personas con discapacidades dislexias de acuerdo a ciertos rangos de edad cómo se observa en la figura 5.

\section{Figura 5 » Grupos Etarios}

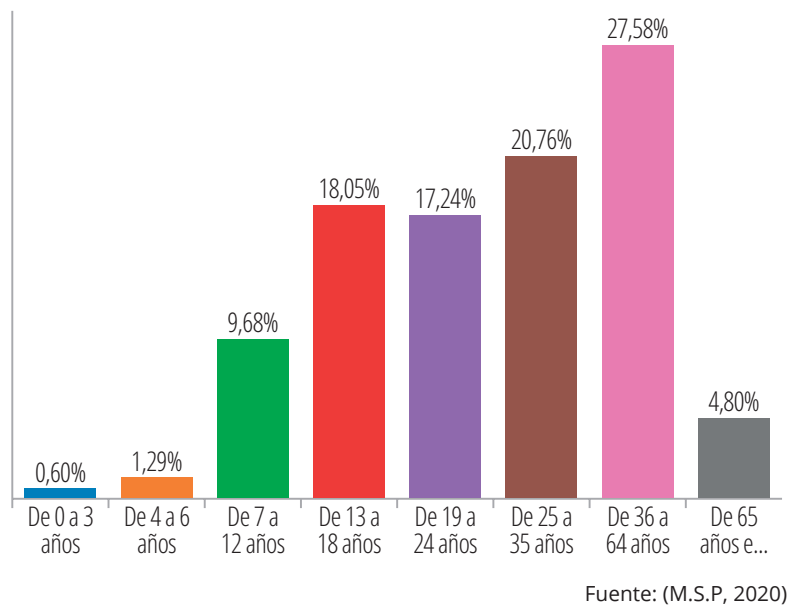

En la actualidad el 1.29\% de las personas con esta discapacidad corresponde a niños de entre 4 a 6 años los mismos que se encuentran en su etapa de preescolar y presentan los siguientes datos de acuerdo a su grado de discapacidad.

Figura 6 » Grado de discapacidad-niños

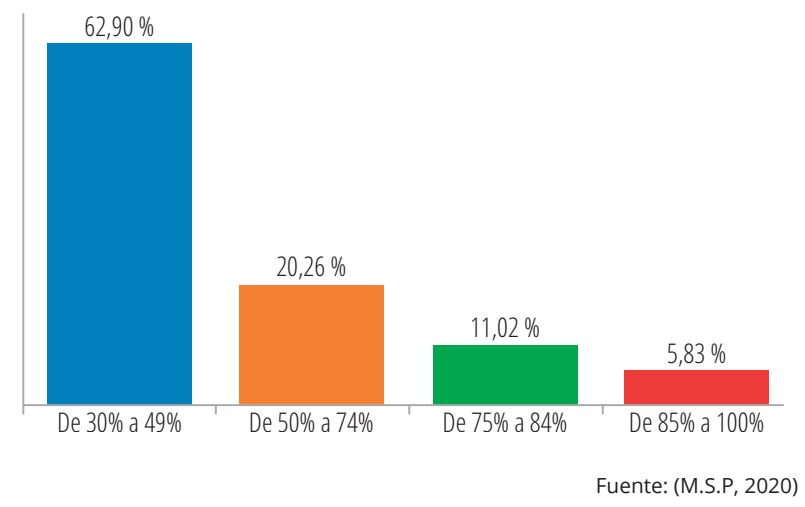

Los diferentes grados de discapacidad presentados en los distintos diagramas permiten evidenciar que la evaluación a los diferentes nichos demográficos es categorizada de acuerdo a las edades de los individuos, teniendo en cuenta que la investigación se centra en niños de preescolar este enfoque tiende a ampliarse debido a la falta de recolección de información en centros educativos y escuelas especiales por ende es necesario la implementación de herramientas digitales que permitan una parcialidad en cuanto a evaluaciones y a la participación activa de padres de familia. 


\section{TÉCNICAS Y MÉTODOS PARA LA DETECCIÓN DE DISLEXIA}

Desde una aproximación conductual, la meta de la evaluación diagnóstica se centra en lo que el niño puede y no puede hacer, en las habilidades que tiene y en las que necesita y en aprovechar esta información para planear las condiciones apropiadas para el aprendizaje. Así, siendo congruentes con los fundamentos de esta aproximación psicológica, se considera entonces que los errores de tipo disléxico que el niño comete no son permanentes, sino que pueden ser modificados a través de la aplicación apropiada de los principios del aprendizaje; en este sentido, se construyó un instrumento con validez de contenido para detectar este tipo de errores (Aragón Borja \& Rodríguez, 2000).

Es muy importante detectar los problemas de dislexia ya que contribuye de manera significativa a la solución de la problemática ya sea en el sentido de lectoescritura, así como en la existencia de dificultades fonológicas (de correcta repetición de determinadas palabras) y las dificultades de pronunciación.

En base a la aplicación de diferentes métodos de evaluación se puede obtener un criterio más específico de los requerimientos para la estructuración de un aplicativo Web, además los diferentes criterios receptados permiten armar un feedback enfocado en los necesidades y requerimientos del sitio de evaluaciones e inclusive de otras herramientas que ayuden a una evaluación más precisa.

La metodología de evaluación consiste en una entrevista inicialmente con el representante del menor de edad y posteriormente con el niño a ser evaluado, esto nos proporciona los elementos suficientes para determinar si es un caso de dislexia o de un niño que presenta únicamente bajo rendimiento en su nivel de escolaridad.

Figura 7 » Proceso de detección y tratamiento de dislexia

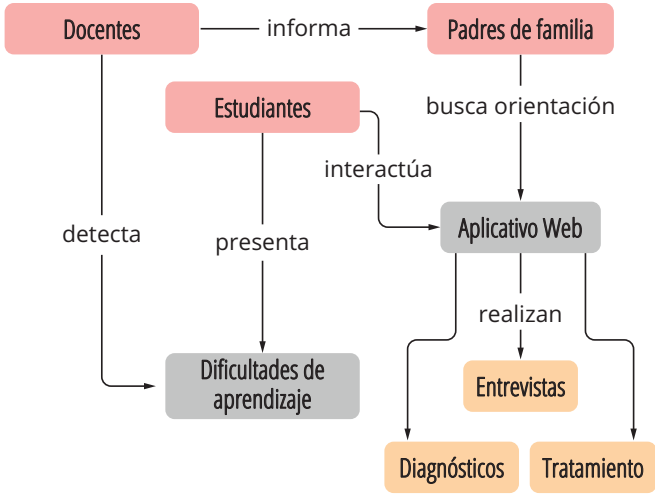

(Rodríguez Martínez et al., n.d.)
Es importante aprovechar esta información ya que mediante la misma se puede usar estrategias especiales para lograr un aprendizaje óptimo del individuo empleando trabajo escolar y herramientas de apoyo útil.

En su mayoría este tipo de pruebas y análisis no cuentan con un software que permita simplificar la labor de los especialistas respecto a la detección y tratamiento de un niño con dislexia por ende todo este procedimiento se lo lleva a cabo por métodos tradicionales ya sean estos libros impresos, material didáctico, ilustraciones entre otros.

\section{MODELO DE PROCESOS PARA EL DISEÑO DE INTERFACES (MPIU - A)}

MPIu+a es una metodología (o modelo) de desarrollo de sistemas interactivos que tiene como meta principal poner al usuario en el centro del desarrollo, buscando maximizar la usabilidad y la experiencia de usuario del sistema final, así como que sea totalmente accesible. (De Jesús et al., n.d.)

Este modelo se establece a partir de la Interacción Persona-Ordenador (IOP) como disciplina y a su vez de la ingeniería de software y de usabilidad con el fin de proceder de una manera organizada y formalizada en el diseño de interfaces de usuario. (Modelo de Proceso de La Ingeniería de La Usabilidad y La Accesibilidad (MPIu+a) | Curso de Interacción Persona-Ordenador, n.d.)

Figura 8 » Esquema del modelo MPlu+a

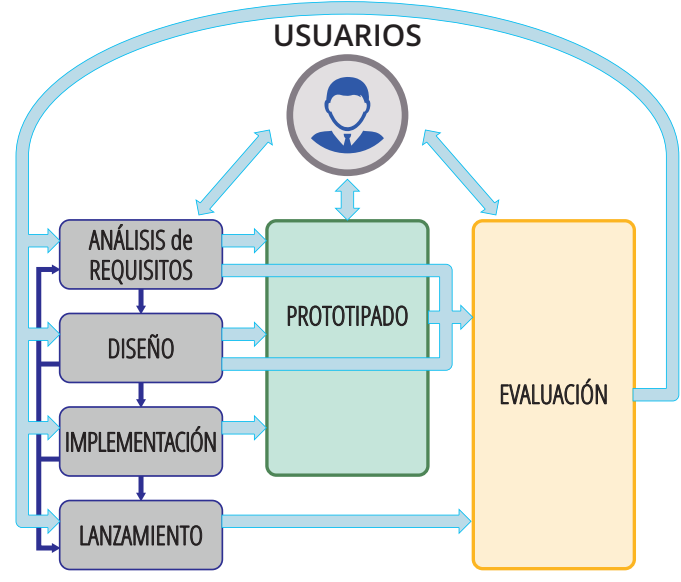

En la figura 7 podemos observar las fases en las que conforman el esquema del modelo MPIu+a y la relación que existe entre ellos. 
MPIu+a al ser un modelo que sigue los principios del diseño centrado en el usuario, tiene las siguientes características principales:

\section{Organización conceptual}

El esquema está organizado en base a una serie de módulos o etapas que determinan la fase de desarrollo en la que nos encontramos y ubica en un nodo concreto la actividad del conocimiento existente en IPO. Esto, en definitiva, no hace más que "poner cada cosa en su sitio», dotando de las pautas a seguir durante el diseño de un sistema interactivo.

En la Ingeniería de la Usabilidad y en la IPO, en general, hay dos conceptos muy importantes que deben realizarse de manera sistemática desde el inicio del desarrollo y no pueden cesar hasta la finalización del sistema: El prototipado y la evaluación.

\section{Tres pilares básicos}

Figura 9 » Pilares del modelo MPlu+a

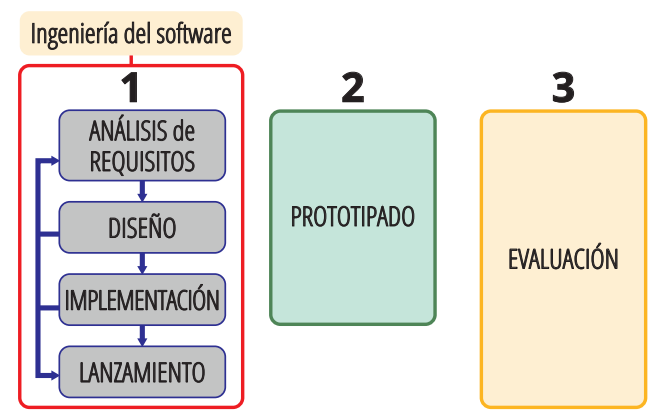

El esquema refleja muy claramente, con una codificación en colores, estos tres conceptos a modo de tres pilares básicos:

- La Ingeniería del Software, en el formato «clásico» de ciclo de vida en cascada iterativo o evolutivo (columna de la izquierda de color azul).

- El prototipado (columna central de color verde), como metodología que engloba técnicas que permitirán la posterior fase de evaluación.

- La evaluación (columna de la derecha, de color amarillo) que engloba y categoriza a los métodos de evaluación existentes.

\section{El usuario}

En los modelos de desarrollo actuales los diseñadores y/o los programadores deciden por los usuarios, escogiendo las metáforas, organizando la información y los enlaces, eligiendo las opciones de los menús, etc. Dichas personas incluso, etiquetan sus aplicaciones como amigables al usuario (con el famoso "user friendly") a pesar de que ningún usuario real haya dado su aprobación a tal característica. Un proceso de Diseño Centrado en el Usuario debe dejar claro de que es así sólo con mirar el esquema la primera vez. Esto es lo que queda reflejado al disponer a éste en la parte central y por encima del resto de etapas todo el modelo de proceso.

Otro aspecto determinante en este modelo de proceso es que se da mucha importancia no sólo a los usuarios, sino también a los implicados (o stakeholders) en cuanto a que son personas que sin ser usuarios directos del sistema su actividad se ve afectada por el mismo. Queda claro, pues, que el usuario está en el centro del desarrollo y en las facetas en las que interviene.

\section{Un método iterativo}

Establecer procesos repetitivos es un aspecto natural que se da en cualquier otro ámbito de ingeniería, sea de la disciplina que sea.

Por poner un ejemplo de otra disciplina, en el mundo de la edificación existe incluso antes de empezar con la excavación del terreno una serie de reuniones (iteraciones) arquitecto-cliente (desarrollador-usuario) para conseguir que el diseño del futuro edificio se adapte a las necesidades de sus inquilinos. Dicho proceso de repetición aplicado a la Ingeniería del Software también existe, aunque suele producirse en la fase final del proceso.

\section{Sencillez}

Mayoritariamente los desarrolladores de sistemas interactivos que pretendemos que la usabilidad sea un factor determinante de los mismos estamos de acuerdo en que sus interfaces, sin perder su capacidad comunicativa y funcional, tienen que ser cuanto más sencillas y simples mejor. Y si estamos de acuerdo con la premisa anterior estaremos igualmente de acuerdo con la idea de que la metodología que les permita llevar a cabo su trabajo de manera más eficiente sea también muy sencilla y simple. 


\section{Adaptado al modelo mental de los equipos multidisciplinares}

El constante contacto con personas procedentes de áreas de conocimiento tan diversas como los componentes del grupo de investigación GRIHO ha servido para constatar la tan referenciada necesidad y a la vez la valiosa aportación que supone trabajar con estos equipos multidisciplinares.

Entre otros aspectos, experimentalmente hemos comprobado que los modelos mentales de las diferentes personas distan mucho entre ellos, hecho que supone que surgen más dificultades de las previstas si los mecanismos de comunicación no son eficientes y las herramientas formales de modelado no son suficientemente simples.

\section{Flexibilidad}

Debe destacarse también que el modelo no tiene ni un sentido lineal ni restrictivo, sino que fomenta la libre aplicación del mismo: Será el equipo de desarrollo (representados normalmente por el responsable del proyecto en desarrollos de envergadura considerable o el diseñador o programador más experimentado en desarrollos menores) junto con los propios requisitos del sistema, las particularidades de los usuarios y los resultados de las diferentes evaluaciones quien marcará cuantas iteraciones deben realizarse, como deben hacerse y el flujo de las acciones a realizar en cada iteración.

\section{SOFTWARE EDUCATIVO PARA EL TRATAMIENTO DE DISLEXIA EN NIÑOS EN ETAPA PRESCOLAR}

Como uno de los objetivos fundamentales de este proyecto es aprovechar el uso de tecnologías agiles existentes ya sea para el desarrollo, así como de la parte investigativa.

Una serie de estudios realizados previo al desarrollo del sistema permite de manera amplia tener un mayor grado de conocimiento acerca de la usabilidad y adaptación que va a tener los niños y niñas al momento de usar este sistema esto con el fin de potenciar las dificultades en el aprendizaje de presentan.

Los principales beneficiarios tanto de los resultados obtenidos de la investigación, así como del sistema a de- sarrollarse son los niños que presentan este tipo de discapacidad así también como los padres de familia o tutores que esté a cargo, resulta además eficiente su implementación en centros educativos para una mejor orientación de los maestros y docentes con la finalidad de llevar una enseñanza paralela conjuntamente con los demás niños.

\section{A. Inteligencia Artificial aplicada a la educación}

Los programas educativos pueden tratar las diferentes materias (matemáticas, idiomas, geografía, dibujo...), de formas muy diversas (a partir de cuestionarios, facilitando una información estructurada a los alumnos, mediante la simulación de fenómenos...) y ofrecer un entorno de trabajo más o menos sensible a las circunstancias de los alumnos y más o menos rico en posibilidades de interacción.(Marqués, n.d.)

La inteligencia artificial parte desde un punto estratégico en el ámbito educativo esto quiere decir que permite un mayor enfoque al desarrollo de actividades que permitan un avance más preciso en la enseñanza.

Por lo tanto, su aplicación y el desarrollo de técnicas que incorporan I.A en el aprendizaje preescolar en especial de niños que presentan dislexia es fundamental para una mayor orientación de los padres y docentes al momento de conocer estos problemas presentes en sus hijos o estudiantes.

Uno de los temas a la que se enfoca la inteligencia artificial es la lectoescritura ya que contiene dos fases fundamentales que son la adquisición y el dominio. En la primera fase, presenta los diferentes niveles conceptuales que desarrolla el niño, desde edades tempranas, en su intento por comprender el lenguaje escrito, mientras que, en la segunda fase, de dominio, se presentan una serie de procesos (perceptivos, léxicos, sintácticos, semánticos) y estrategias (metacognitivas, inferenciales, etc.) que determinan el nivel de literacia o dominio del sistema de escritura. (Montealegre, n.d.)

Centrándonos en estos conceptos podemos decir que su inmediata evaluación permite tener un anticipado resultado respecto a si el niño presenta o no dislexia. 
El desarrollo de chatsbots ha sido una de las tecnologías que han venido en auge estos últimos tiempos ya que su fácil interacción con los usuarios permite obtener criterios y comentarios positivos para su mejora continua e investigación (Giraldo De La et al., 2017)

Por ello el uso de chatbot en un sistema de detección de dislexia permite a los niños y niñas una orientación respecto a las aptitudes que deben de realizar en el sistema.

B. Metodología de Desarrollo de Software Prototipo

En la elaboración del software se utilizará la metodología de desarrollo Prototipo, debido a que esta metodología incorpora componentes del producto real, esto quiere decir que será una representación del sistema, aunque no es un sistema completo, posee las características del sistema final o parte de ellas.

Su implementación inicialmente se basa en la definición de los objetivos globales para el software para luego pasar a identificar los requisitos conocidos y las tareas del esquema en donde es necesariamente más definición.

Entonces se plantea con rapidez una iteración de construcción de prototipos y se presenta el modelo.

Figura 9 » Pilares del modelo MPlu+a

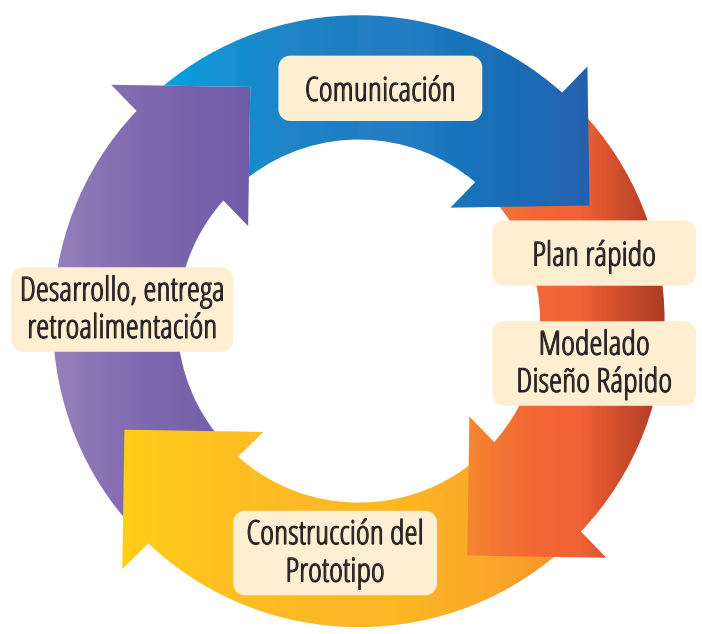

Esta metodología se define en diferentes fases que son:

A. Investigación preliminar

En esta etapa es esencial determinar el problema y su ámbito, la importancia y los efectos potenciales que tendrán sobre la organización.

B. Definición de los requerimientos del sistema

Esta etapa es donde se determina los requerimientos que los usuarios tienen en relación al proyecto que se está deseando implementar.

\section{Análisis grueso y especificación}

En esta fase se busca desarrollar un diseño básico para el prototipo inicial.

\section{Diseño y construcción}

Es donde se consigue un prototipo inicial, aquí el desarrollador debe concentrarse en construir un sistema con la máxima funcionalidad.

\section{E. Evaluación}

En esta etapa es obtener por parte de los usuarios la especificación de los requerimientos adicionales del sistema y verificar que el prototipo desarrollado haya sido en concordancia con la definición de los requerimientos del sistema.

\section{F. Modificación}

Se da cuando la definición de requerimientos del sistema es alterada en la etapa de evaluación. El desarrollador entonces debe de modificar el prototipo de acuerdo a los comentarios hechos por los usuarios.

\section{G. Termino}

Una vez que se ha desarrollado un prototipo estable y completo, es necesario ponerse de acuerdo en relación a aspectos de calidad y de representación del sistema. (Valerio, n.d.)

\section{CONCLUSIONES}

El desarrollo de software educativo enfocado a niños y niñas que presentan una discapacidad dislexia es un punto de partida hacia el desarrollo e innovación en otras áreas de la educación, el uso de inteligencia artificial para reforzar estos trabajos permite una mayor precisión en cuanto a resultados. 
La innovación tecnológica seguida de técnicas educativas para el aprendizaje requiere de una investigación minuciosa por ello el plantear modelos y obtener datos que ayuden al posterior desarrollo del sistema es fundamental, de esta forma se obtiene un software más relacionado con los usuarios finales, las instituciones educativas deben de apuntar a innovación tecnológica no solo aplicada a niños sanos sino a aquellos que presentan un rato de discapacidad ya sea de cualquier índole.

\section{BIBLIOGRAFÍA}

Aragón Borja, L. E., \& Rodríguez, A. S. (2000). Análisis cualitativo de un instrumento para detectar errores de tipo disléxico (IDETID-LEA).

De Jesús, M., Quintero, D., Fuentes, N. Q., \& Rodríguez Martínez, K. (n.d.). Guía de Estilo para el Diseño de Interfaces de Software Educativos para el Apoyo a la Reeducación de los Niños con Dislexia.

Estadísticas de Discapacidad - Consejo Nacional para la Igualdad de Discapacidades. (n.d.). Retrieved September 23, 2020, from https://www.consejodiscapacidades.gob.ec/estadisticas-de-discapacidad/

Giraldo De La, I., León Rodríguez, C., Silvia, L., \& Brito, M. V. (2017). La inteligencia artificial en la educación superior. Oportunidades y amenazas Artificial intelligence in higher education. Opportunities and threats. INNOVA Research Journal, 2(8), 412-422. https://doi.org/10.33890/innova.v2.n8.1.2017.399

Marquès, P. (n.d.). El software educativo.

Modelo de Proceso de la Ingeniería de la Usabilidad y la Accesibilidad (MPIu+a) | Curso de Interacción Persona-Ordenador. (n.d.). Retrieved September 23, 2020, from https://mpiua.invid.udl.cat/fases-mpiua/

Montealegre, R. (n.d.). DESARROLLO DE LA LECTOESCRITURA: ADQUISICIÓN Y DOMINIO 1 DEVELOPMENT OF READING/WRITING SKILLS: ACQUISITION AND DOMINION.

RodríguezMartínez,K., De Jesús, M.,Quintero,D.,\& Fuentes, N. Q. (n.d.). Desarrollo de un Software Educativo para el Tratamiento de los Niños con Dislexia en Panamá aplicando el MPIu+a para el Diseño de las Interfaces de Usuario.
Valerio, K. (n.d.). metodologia de prototipos. Retrieved September 23, 2020, from https://es.slideshare. net/KeinerValerio/metodologia-de-prototipos

Vallejo, A. (n.d.). UNIVERSIDAD DEL AZUAY FACULTAD DE FILOSOFIA, LETRAS Y CIENCIAS DE LA EDUCACIÓN ESCUELA DE PSICOLOGIA EDUCATIVA Y TERAPEUTICA. 